\title{
Discretized aperture mapping with a micro-lenses array for interferometric direct imaging
}

\author{
Fabien Patru ${ }^{a}$, Jacopo Antichi ${ }^{a}$, Dimitri Mawet ${ }^{b}$, Laurent Jolissaint $^{c}$, Marcel Carbillet ${ }^{d}$, Julien Milli ${ }^{b}$, \\ Julien Girard ${ }^{b}$, Patrick Rabou ${ }^{e}$, Enrico Giro $^{f}$ and Denis Mourard ${ }^{g}$ \\ ${ }^{a}$ Osservatorio Astrofisico di Arcetri, 5 Largo Enrico Fermi, 50125, Firenze, Italia \\ ${ }^{b}$ European Southern Observatory, Alonso de Cordova 3107, 19001, Vitacura, Chile \\ ${ }^{c}$ Haute Ecole d'Ingenierie et de Gestion du Canton de Vaud, Yverdon-les-Bains, Switzerland ${ }^{d}$ \\ Observatoire de la Cote d'Azur, Parc Valrose, 06108, Nice, France \\ ${ }^{e}$ Institut de Planetologie et d'Astrophysique, 414 Rue de la piscine, 38041, Grenoble, France \\ ${ }^{f}$ Osservatorio Astronomico di Padova, Vicolo dell'Osservatorio 5, 35122, Padova, Italia \\ $g$ Observatoire de la Cote d'Azur, 2130 Boulevard de l'Observatoire, 06304, Nice, France
}

\begin{abstract}
Discretized Aperture Mapping (DAM) appears as an original filtering technique easy to play with existing adaptive optics (AO) systems. In its essential DAM operates as an optical passive filter removing part of the phase residuals in the wavefront without introducing any difficult-to-align component in the Fourier conjugate of the entrance pupil plane. DAM reveals as a new interferometric technique combined with spatial filtering allowing direct imaging over a narrow field of view (FOV). In fact, the entrance pupil of a single telescope is divided into many sub-pupils so that the residual phase in each sub-pupil is filtered up to the DAM cut-off frequency. DAM enables to smooth the small scale wavefront defects which correspond to high spatial frequencies in the pupil plane and to low angular frequencies in the image plane. Close to the AO Nyquist frequency, such pupil plane spatial frequencies are not well measured by the wavefront sensor (WFS) due to aliasing. Once bigger than the AO Nyquist frequency, they are no more measured by the WFS due to the fitting limit responsible for the narrow AO FOV. The corresponding image plane angular frequencies are not transmitted by DAM and are useless to image small FOVs, as stated by interferometry. That is why AO and DAM are complementary assuming that the DAM cut-off frequency is equal to the AO Nyquist frequency. Here we describe the imaging capabilities when DAM is placed downstream an AO system, over a convenient pupil which precedes the scientific detector. We show firstly that the imaging properties are preserved on a narrow FOV allowing direct imaging throughout interferometry. Then we show how the residual pupil plane spatial frequencies bigger than the AO Nyquist one are filtered out, as well as the residual halo in the image is dimmed.
\end{abstract}

Keywords: adaptive optique, aliasing, spatial filtering, micro-lenses array

\section{INTRODUCTION}

\subsection{The DAM concept}

$\mathrm{DAM}^{1,2}$ is an optical passive filter providing a discretized wavefront. The pupil discretization sub-divides the full aperture in many discret sub-apertures in the pupil plane. Each discretized sub-aperture is spatially or modally filtered to bring them coherent in phase, i.e. to remove out the phase aberrations within each sub-aperture. The differential phase between each pair of sub-apertures is preserved, i.e. the high angular resolution information is transmitted through DAM.

This new optical process is called Discretized Aperture Mapping. The terms densified or dense aperture previously used $^{1,2}$ are related to less significant interferometric considerations and are now replaced by the term discretized aperture.

The term aperture mapping includes DAM in the pupil mapping techniques used in coronographic imaging and in interferometric imaging. Continous pupil mapping - PIAA - geometrically rearranges the light in the pupil plane without

Further author information: (Send correspondence to Fabien Patru)

Fabien Patru: E-mail: fabienpatru@ gmail.com

Jacopo Antichi: E-mail: antichj@gmail.com

Adaptive Optics Systems IV, edited by Enrico Marchetti, Laird M. Close,

Jean-Pierre Véran, Proc. of SPIE Vol. 9148, 91485P - (c) 2014 SPIE

CCC code: $0277-786 \mathrm{X} / 14 / \$ 18 \cdot$ doi: $10.1117 / 12.2058594$

Proc. of SPIE Vol. 9148 91485P-1 
introducing phase aberrations, ${ }^{3,4}$ i.e. the output electric field is amplitude-modified but still flat in phase (at least for onaxis sources). In the same way, discretized pupil mapping - DAM - modifies the shape in amplitude of the sub-aperture thanks to spatial filtering, but preserves the differential phase - piston - between the sub-apertures. ${ }^{2}$ If PIAA is used to apodized the pupil amplitude by modulating the phase, DAM is used to smooth the pupil phase by modulating the pupil amplitude. However, both rely on the same optical approach by modifying the phase thanks to amplitude modulation, either by rearranging the light (PIAA) or by filtering the light (DAM).

The pupil discretization is similar in few aspects with the pupil mapping, ${ }^{2,3,5}$ the pupil remapping, ${ }^{1,6}$ the pupil densification, ${ }^{7-10}$ the pupil segmentation ${ }^{11}$ as well as with the well-known diffraction gratting. ${ }^{7}$ Finally, DAM can be seen as part of the interferometric direct imaging (IDI) techniques suitable for high-contrast imaging applications using adaptive optics (AO).

\subsection{The BIGRE micro-lenses array}

The optical discretization of the telescope aperture is achievable thanks to new integrated components developped in the fields of optical stellar interferometry and spatial or modal filtering techniques. In fact, modal filtering is usually performed on a multi-aperture system by a single-mode (SM) waveguide array, such as single-mode fiber array ${ }^{12-15}$ and integrated guided optics, like planar optical components ${ }^{16,17}$ or three-dimensional photonic circuits. ${ }^{18}$

Nonetheless, spatial filtering can also be achieved by suitable mask-holes in the intermediate focal plane of two microlenses arrays, or in the pupil plane by an afocal double micro-lenses array called BIGRE ${ }^{19}$ and fine tuned as a spatial filter for DAM. ${ }^{20}$ We study here the case of BIGRE-DAM, which appears as the great poor-man technical solution (small, simple, stable) easily integrable in present day and coming AO systems devoted to direct imaging of exoplanets (e.g. VLT/SPHERE, E-ELT/PCS).

\subsection{The AO \& DAM combination}

The pupil discretization is a new optical process suitable with present natural guide star (NGS) AO systems. The discretized aperture provides the same imaging properties than the full AO-corrected aperture on a narrow FOV. The main benefit is the DAM filtering property of AO residual aberrations, but the main drawback is a global flux loss of few magnitudes.

We present in this paper an application of BIGRE-DAM with a 8m-telescope equipped with a classical AO system. AOcorrected wavefronts have been generated with the software PAOLA. ${ }^{21}$ We assume low atmospheric turbulence (seeing of 0.85 , coherence time of $3 m s$ ) allowing a high level of AO compensation with a bright NGS of magnitude 10. In such case, AO are slightly affected by the WFS read-out noise error, the servo-lag error, the anisplanatism error, but are still dominated by fundamental $\mathrm{AO}$ limits that are mainly the fitting limit and the aliasing effect. ${ }^{21}$

We consider the monochromatic wavelength $1.65 \mu \mathrm{m}$. We establish that that the optical train and the BIGRE microlenses array are out of any optical aberration. We show up with numerical simulations the imaging and filtering properties of DAM, when it is placed downstream an AO system, over a convenient pupil which precedes the scientific detector.

\section{DAM IMAGING AND FILTERING PROPERTIES}

\subsection{DAM wavefront (amplitude and phase)}

The wavefront of the entrance aperture of the telescope is discretized by DAM. DAM subdivides the aperture of diameter $D$ in many coherent sub-apertures of diameter $d$, so that the number of sub-apertures along the telescope diameter is equal to $N=D / d$. Coherent means that spatial or modal filtering is applied on each sub-aperture and that the residual phase of each of the sub-pupil is filtered out. Modal filtering allows a perfect filtering, since a unique flat phase is transmitted by the single-mode waveguide. On the other hand, spatial filtering is less efficient, since low orders like tip-tilt are transmitted due to the fact that a mask-hole or a BIGRE component are still multi-mode. The discretized wavefront phase has been smoothed on each sub-aperture in the case of BIGRE spatial filtering, as shown in figures 3 and 4 . The discretized wavefront amplitude has a gaussian distribution on each sub-aperture, as shown in figures 1 and 2.

We assume here that the DAM cut-off frequency is equal to the AO Nyquist frequency (see Sect. 2.4), so that the pitch $d$ of the micro-lenses of a BIGRE-DAM matches the pitch between the actuators of the AO deformable mirror (DM). Moreover the pitch of the Shack-Hartmann (SH-WFS) micro-lenses array is equal to the pitch of the BIGRE-DAM microlenses array. We finally assume that the DM actuators, the SH-WFS micro-lenses and the BIGRE-DAM micro-lenses are 

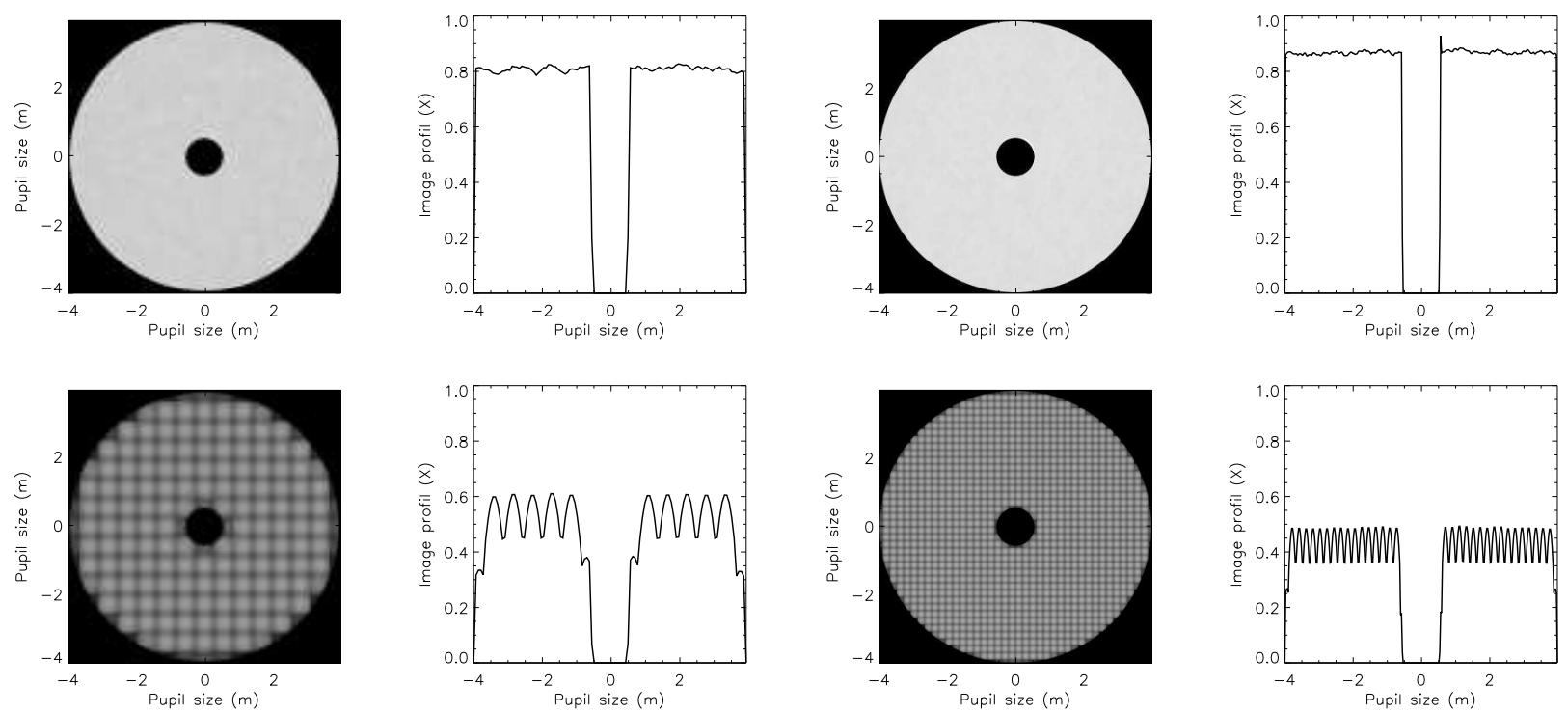

Figure 1. Pupil amplitude (2D, X profil) without DAM (top) and with DAM (bottom) using AO of $15 \times 15$ (left) and $40 \times 40$ (right) actuators.
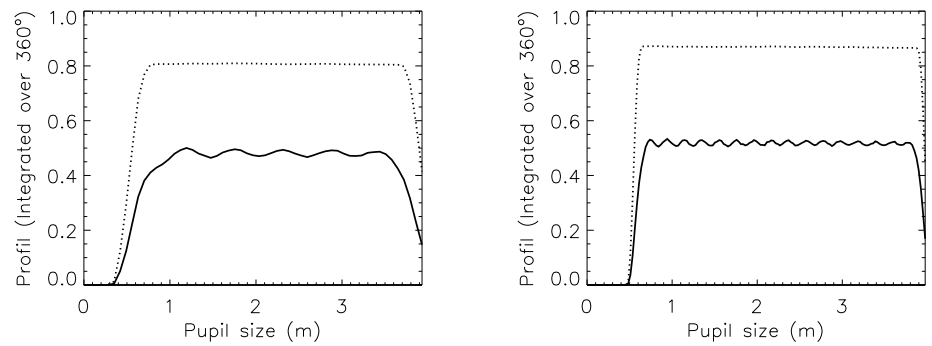

Figure 2. Pupil amplitude (profil averaged over $360^{\circ}$ ) without DAM (dotted line) and with DAM (solid line) using AO of $15 \times 15$ (left) and $40 \times 40$ (right) actuators.

homothetics and distributed over a squared grid. Thus, the number of DM actuators, the number of sub-apertures on the WFS and on DAM are all equal. We consider the AO sampling of $N \times N=15 \times 15$ and $N \times N=40 \times 40$, corresponding respectively to a sub-aperture diameter $d=50 \mathrm{~cm}$ and $d=20 \mathrm{~cm}$ over a telescope diameter $D=8 \mathrm{~m}$. These cases mimic the AO sampling of NAOS ${ }^{22}$ and SPHERE $^{23}$ at the VLT.

We assume also that the BIGRE has a high level of spatial filtering with a poor flux transmission. The equivalent mask-hole in each sub-aperture is chosen with a small size approximatelly equal to the Full Width at Half Maximum $(F W H M=\lambda / d)$ of the sub-image. $F W H M=\lambda / d$ is 2.44 times lower than the width of the Airy disk containing $80 \%$ of the flux. Such level of spatial filtering increases the number of filtered aberrations of low-orders, but at the cost of flux loss. The flux throughput is lower than $50 \%$ here in good atmospheric conditions, but it can drop rapidely down to few pourcents in worse conditions.

\subsection{DAM point spread function}

The point spread function (PSF) is defined as the intensity distribution in the image of a point source, equal to the Fourier transform of the autocorrelation of the aperture transmission (in phase and in amplitude). Each defect in the pupil plane can damage the diffrative pattern in the image plane. The DAM PSF is shown in figures 5 and 6 . The angular resolution is imposed by the telescope diameter $D$ (maximum interferometric baseline) and by the wavelength $\lambda$, so that a resolution element (resel) is equal to resel $=\lambda / D$. The useful FOV is imposed by the diffraction envelope of a sub-aperture of diameter $d$ (minimum interferometric baseline), so that $F O V=\lambda / d$. The FOV in number of resolution elements (resel) 

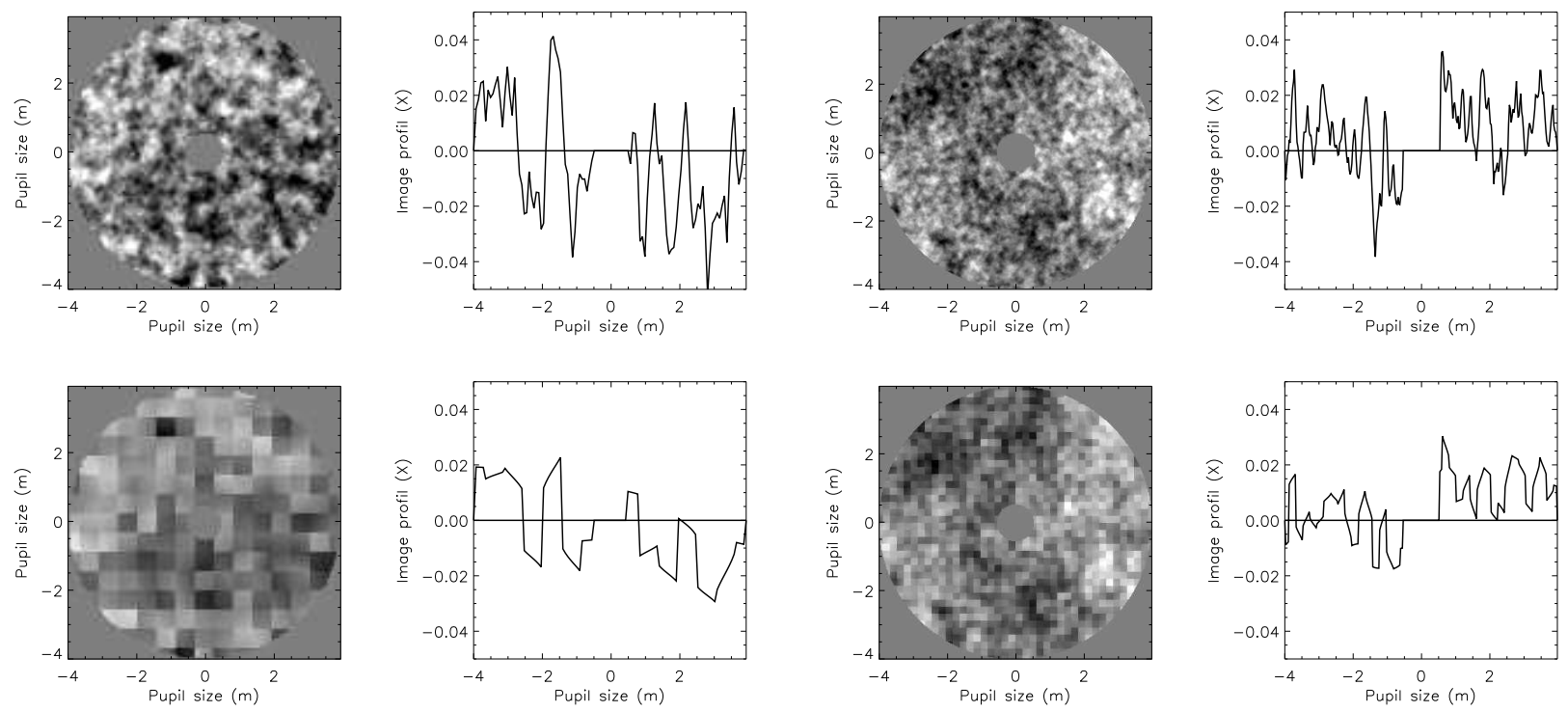

Figure 3. Pupil phase (2D, X profil) without DAM (top) and with DAM (bottom) using AO of $15 \times 15$ (left) and $40 \times 40$ (right) actuators.
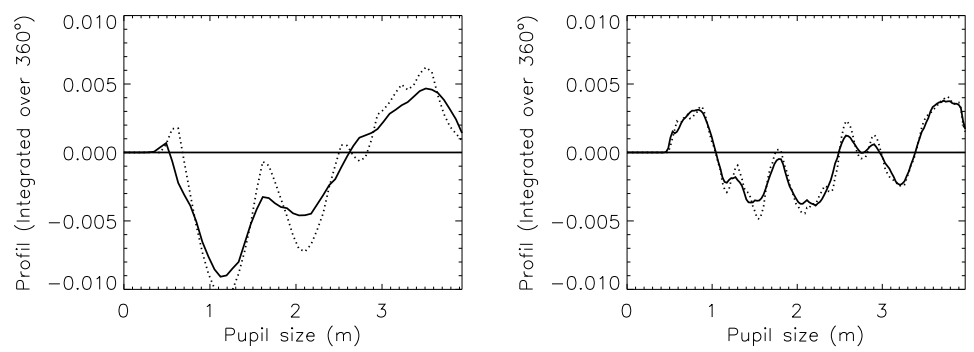

Figure 4. Pupil phase (profil averaged over $360^{\circ}$ ) without DAM (dotted line) and with DAM (solid line) using AO of $15 \times 15$ (left) and $40 \times 40$ (right) actuators.

is equal to the number of sub-apertures along the telescope diameter, i.e. $F O V($ resel $)=D / d$. DAM removes part of the AO-halo in the PSF and enhances the ultimate contrast, especially at the edge of the useful FOV provided by AO, where aliasing is more significant with a SH-WFS.

\subsection{DAM optical transfert function}

The optical transfert function (OTF) is defined as the Fourier transform of the PSF. The DAM OTF is shown in figures 7 and 8. The perfect OTF has a triangular shape, meaning that all the angular frequencies in the focal plane are probed on the sky and are transmitted homogeneously through either the full aperture or the discretized aperture. The turbulent OTF of the DAM aperture is lower in amplitude than the full aperture, due to the absolute flux loss induced by spatial filtering. The main drawback of DAM is a moderate flux throughput, which may restrain the sky coverage for scientific applications. However, the shape of the DAM OTF remains almost triangular, meaning that the relative flux loss between the image plane angular frequencies remains inversely proportional to the angular frequency. DAM coupled with AO provides an homogeneous transmission of the angular frequencies sampled on the sky.

DAM preserves the high angular imaging properties on a narrow FOV by transmitting the large scale structures in the pupil plane which are sampled by each pair of sub-apertures and which correspond to the high image plane angular frequencies. According to interferometry, ${ }^{8}$ high angular frequencies are enough to image a narrow FOV, whereas low angular frequencies are useless close to the central axis. The FOV is limited by the smallest image plane angular frequency sampled on the sky and transmitted by the AO \& DAM system. 

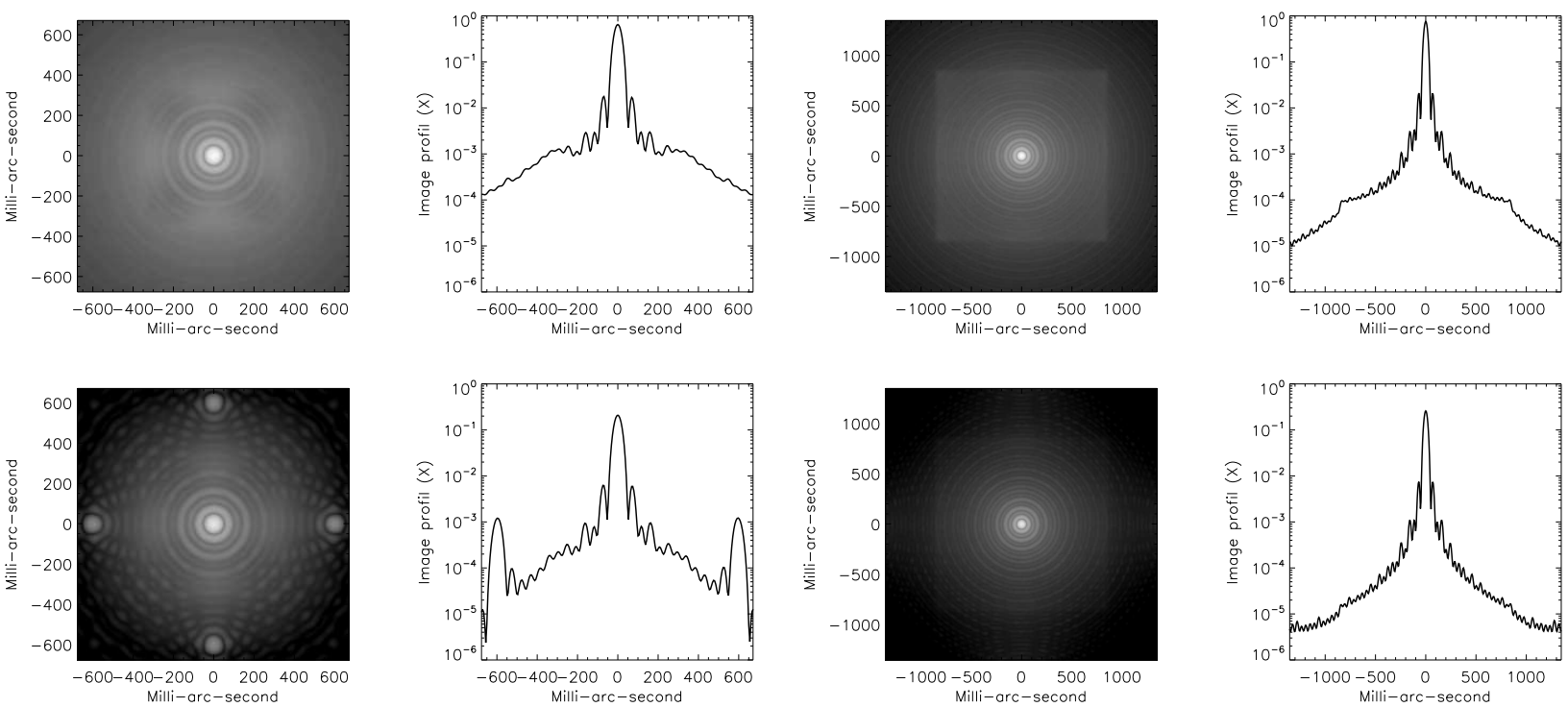

Figure 5. Point spread function (2D, X profil) without DAM (top) and with DAM (bottom) using AO of $15 \times 15$ (left) and $40 \times 40$ (right) actuators. In $\mathrm{H}$ band at $1.65 \mu \mathrm{m}$, the FOV is respectively equal to 0.8 as and to 1.6 as and the resolution power is equal to $40 \mathrm{mas}$ with a $8 \mathrm{~m}$-telescope. The $40 \times 40 \mathrm{PSF}$ has been enlarged twice compared with the $15 \times 15 \mathrm{PSF}$.
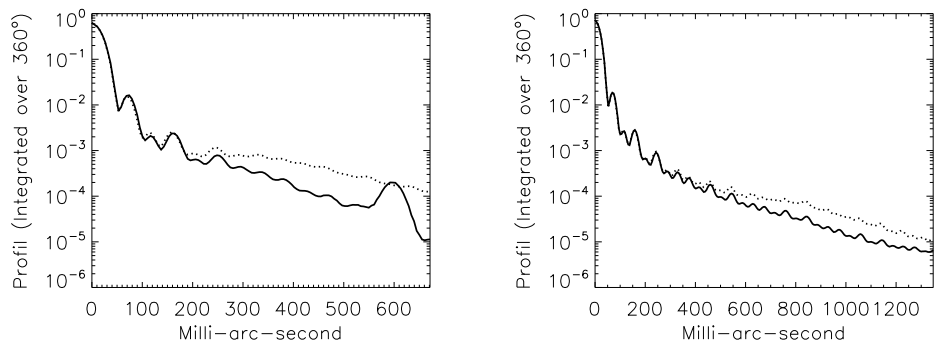

Figure 6. Point spread function (profil averaged over $360^{\circ}$ ) without DAM (dotted line) and with DAM (solid line) using AO of $15 \times 15$ (left) and $40 \times 40$ (right) actuators. The PSFs have been normalized to the maximum value of the diffraction limited PSF, so that the flux loss due to DAM is not taken into account, but the relative flux of the diffractive pattern can be compared. The $40 \times 40$ PSF has been enlarged twice compared with the $15 \times 15 \mathrm{PSF}$, so that the angular resolution appears two times lower, but the edge of the useful FOV remains visible.

DAM improves the high contrast imaging properties by spatially filtering the small scale structures in the pupil plane which are smaller than the sub-aperture width and which correspond to the low image plane angular frequencies. The central peak of radius $1 / d$, corresponding to incoherent energy at the entrance pupil position, dominates the image with the full aperture (without DAM). This is due mainly to AO fitting error which limits the FOV extension in the focal plane. The incoherent energy in the central peak is strongly attenuated when using DAM.

Assuming that the DAM cut-off frequency is equal to the AO Nyquist frequency, it comes: $v=d / \lambda=v_{D A M}=v_{A O}=$ $v_{D M}=v_{W F S}$. This means that DAM does not introduce FOV limitations already imposed by the AO fitting limit. AO corrects the high image plane angular frequencies probed on the sky, whereas DAM filters out the low image plane angular frequencies useless on the narrow FOV provided by AO.

\subsection{DAM power spectral density}

The phase spatial power spectral density (PSD) is equal to the statistic average of the Fourier transforms of the residual AO-compensated phase. The DAM PSD is shown in figures 9 and 10. DAM allows to filter the wavefront defects which are smaller than the sub-aperture diameter and which correspond to the high pupil plane spatial frequencies. DAM is a 

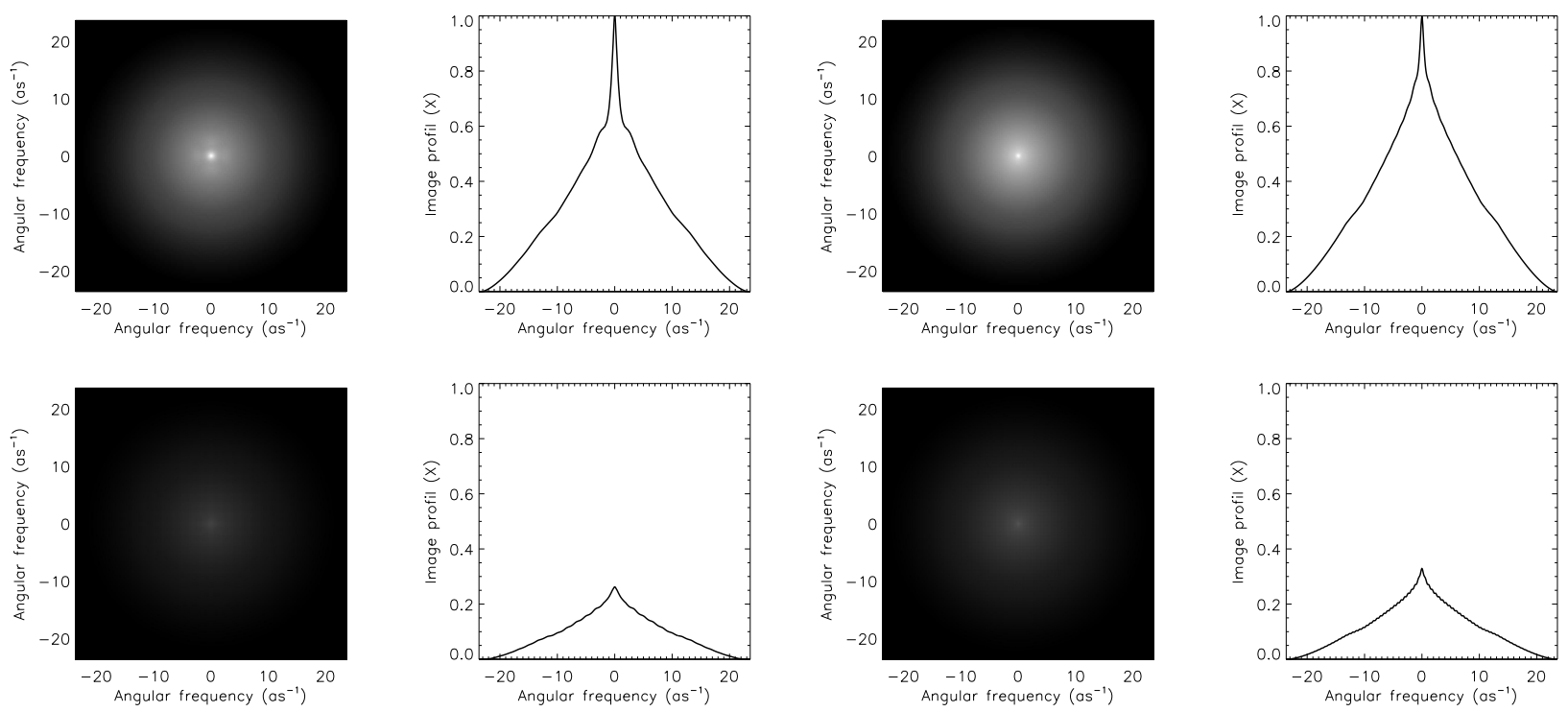

Figure 7. Optical transfert function amplitude (2D, X profil) without DAM (top) and with DAM (bottom) using AO of $15 \times 15$ (left) and $40 \times 40$ (right) actuators. In $\mathrm{H}$ band at $1.65 \mu \mathrm{m}$, the lowest image plane angular frequency is respectively equal to $1.8 \mathrm{as}^{-1}$ and to $0.6 \mathrm{as}^{-1}$ and the highest image plane angular frequency is equal to $24 \mathrm{as}^{-1}$.
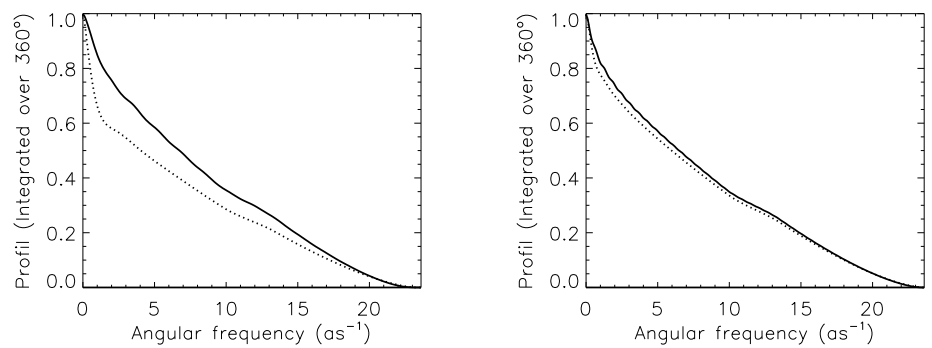

Figure 8. Optical transfert function amplitude (profil averaged over $360^{\circ}$ ) without DAM (dotted line) and with DAM (solid line) using $\mathrm{AO}$ of $15 \times 15$ (left) and $40 \times 40$ (right) actuators. The OTFs have been normalized with their maximum value equal to 1 , so that the cases with and without DAM can be compared.

low-pass spatial frequency filter in the pupil plane, which transmits all the low spatial frequencies up to the DAM cut-off frequency $f=1 / 2 d$ and removes out the high spatial frequencies above $f$.

$\mathrm{AO}$ enables to correct the wavefront defects which are larger than twice the pitch of the DM and which correspond to the low pupil plane spatial frequencies. AO is by definition a high-pass spatial frequency filter in the pupil plane, which attenuates the low spatial frequency phase residuals up to the AO Nyquist frequency $f_{A O}=\min \left(f_{D M}, f_{W F S}\right)$. However, the spatial frequencies higher than $f_{A O}$ are not seen by the WFS and are transmitted through the AO system. This is due mainly to AO fitting error, which occurs far from the axis and imposes the limits of the useful FOV (Nyquist theorem). The spatial frequencies lower than $f_{A O}$ but close to $f_{A O}$ are not well corrected by AO. This is due mainly to AO aliasing error, because high spatial frequencies above $f_{A O}$ are seen by the WFS as low spatial frequencies below $f_{A O}$. Consequently, the DM tries to correct residual phases which do not exist.

Assuming that the DAM cut-off frequency is equal to the AO Nyquist frequency, it comes: $f=1 / 2 d=f_{D A M}=f_{A O}=$ $f_{D M}=f_{W F S}$. DAM removes both the $\mathrm{AO}$ fitting error and the $\mathrm{AO}$ aliasing error. The result is that DAM can improve the image quality (Strehl gain) and the image stability (aliasing error reduction) of an AO system. AO corrects the low pupil plane spatial frequency phase defects, whereas DAM filters out the high pupil plane spatial frequencies unseen or not well corrected by AO. 

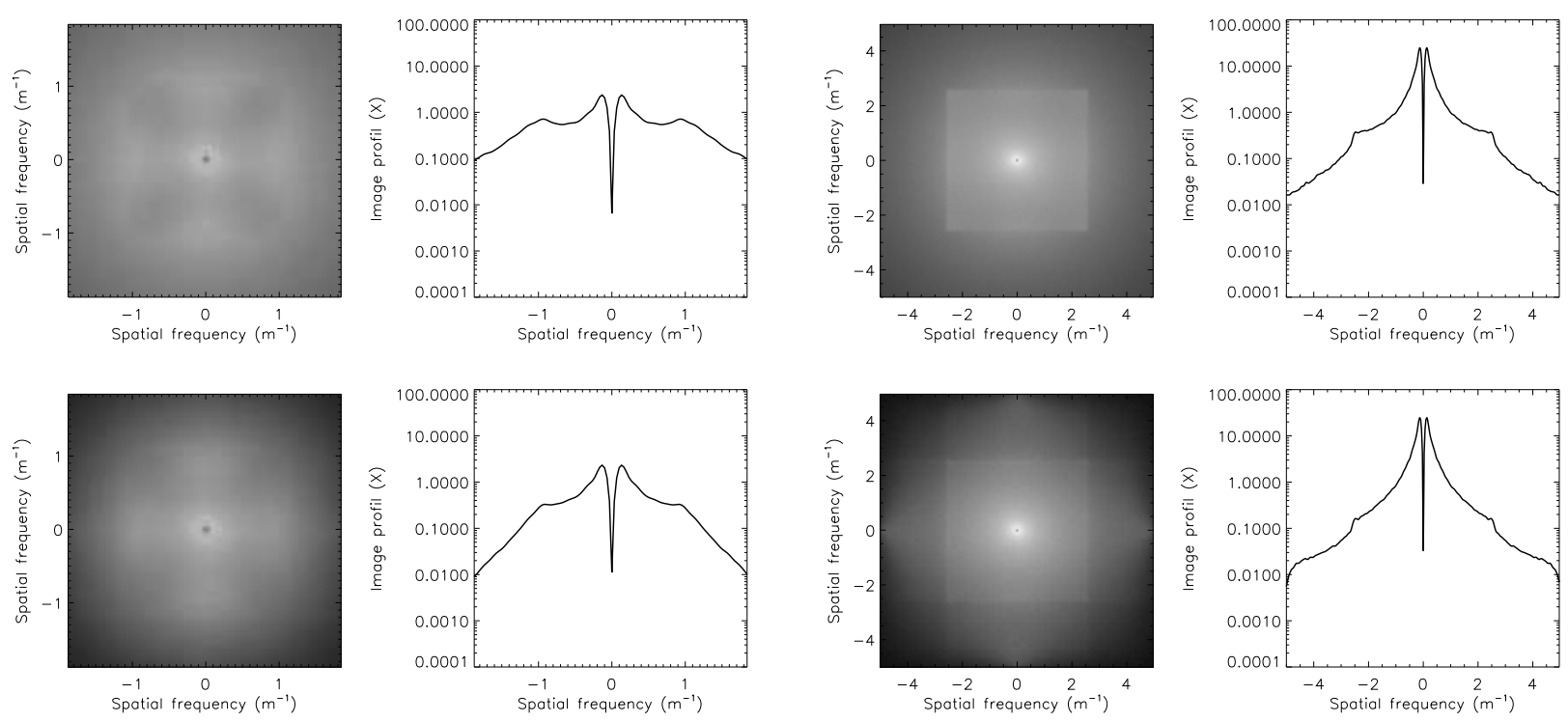

Figure 9. Phase power spectral density (2D, X profil) without DAM (top) and with DAM (bottom) using AO of $15 \times 15$ (left) and $40 \times 40$ (right) actuators. In $\mathrm{H}$ band at $1.65 \mu \mathrm{m}$, the cut-off pupil plane spatial frequency - Nyquist frequency - is respectively equal to $2.5 \mathrm{~m}^{-1}$ and to $1 \mathrm{~m}^{-1}$
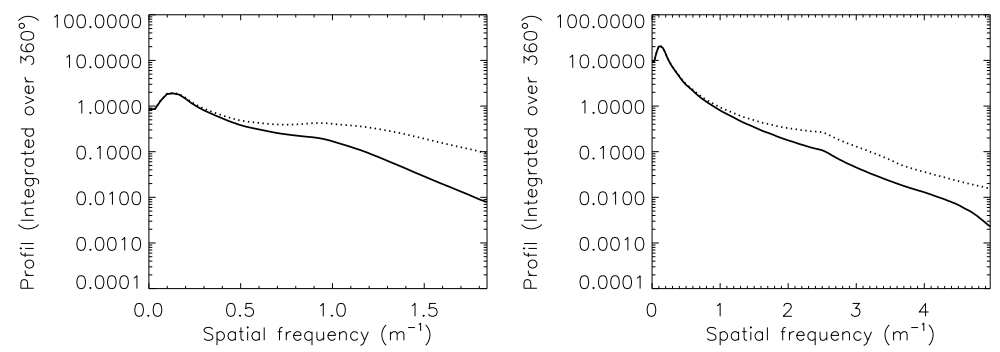

Figure 10. Phase power spectral density (profil averaged over $360^{\circ}$ ) without DAM (dotted line) and with DAM (solid line) using AO of $15 \times 15$ (left) and $40 \times 40$ (right) actuators.

\section{CONCLUSION AND PERSPECTIVE}

These first simulations show up the interest of using DAM in high-contrast imaging. DAM is an efficient and passive optical filter which fits with the needs of high contrast imaging techniques in astronomy, hopefully not only with classical AO systems, as shown here with DAM applied to SH-WFS, but also with other more sophisticated AO systems (e.g. MCAO) or WFS (e.g. Pyramid). Moreover, DAM is versatile and compatible with many observational modes (e.g. coronography or differential imaging). Finally, BIGRE-DAM is an easy to plug-in device, which can be integrated in the current and coming $\mathrm{AO}$ systems as an upgrade.

\section{REFERENCES}

[1] Patru, F., Antichi, J., and Girard, J., "Direct imaging with a dense aperture masking in comparison with a telescope or a hypertelelescope," in [SPIE], 8172 (2011).

[2] Patru, F., Antichi, J., Girard, J., and Jolissaint, L., "Diffraction limited imaging with Dense Aperture Mapping," in [Second International Conference on Adaptive Optics for Extremely Large Telescopes. ], (2011).

[3] Guyon, O., "Phase-induced amplitude apodization of telescope pupils for extrasolar terrestrial planet imaging," AEA 404, 379-387 (2003). 
[4] Guyon, O., "Pupil Remapping Techniques for High Dynamical Range Imaging," in [EAS Publications Series], 12, 11-20 (2004).

[5] Vanderbei, R. J. and Traub, W. A., "Pupil Mapping in Two Dimensions for High-Contrast Imaging,” ApJ 626, 1079_ 1090 (2005).

[6] Perrin, G., Lacour, S., Woillez, J., and Thiébaut, É., "High dynamic range imaging by pupil single-mode filtering and remapping," MNRAS 373, 747-751 (2006).

[7] Labeyrie, A., "Resolved imaging of extra-solar planets with future 10-100km optical interferometric arrays.," AE A AS 118, 517-524 (1996).

[8] Lardière, O., Martinache, F., and Patru, F., "Direct imaging with highly diluted apertures - I. Field-of-view limitations," MNRAS 375, 977-988 (2007).

[9] Patru, F., Tarmoul, N., Mourard, D., and Lardière, O., "Direct imaging with highly diluted apertures - II. Properties of the point spread function of a hypertelescope," MNRAS 395, 2363-2372 (2009).

[10] Patru, F., Chiavassa, A., Mourard, D., and Tarmoul, N., "Direct imaging with a hypertelescope of red supergiant stellar surfaces," in [SPIE], SPIE $\mathbf{7 7 3 4}$ (2010).

[11] Yaitskova, N., Dohlen, K., and Dierickx, P., "Analytical study of diffraction effects in extremely large segmented telescopes," Journal of the Optical Society of America A 20, 1563-1575 (2003).

[12] Shao, M., "Hubble Extra Solar Planet Interferometer," in [Space Astronomical Telescopes and Instruments], 1494, 347-356 (1991).

[13] Mennesson, B. P., Shao, M., Levine, B. M., Wallace, J. K., Liu, D. T., Serabyn, E., Unwin, S. C., and Beichman, C. A., "Optical Planet Discoverer: how to turn a 1.5-m class space telescope into a powerful exo-planetary systems imager," in [High-Contrast Imaging for Exo-Planet Detection. ], 4860, 32-44 (2003).

[14] Patru, F., Mourard, D., Lardière, O., and Lagarde, S., "Optimization of the direct imaging properties of an opticalfibred long baseline interferometer," MNRAS 376, 1047-1053 (2007).

[15] Patru, F., Mourard, D., Clausse, J., Delage, L., Reynaud, F., Dubreuil, M., Bonneau, D., Bosio, S., Bresson, Y., Hugues, Y., Lardière, O., and Roussel, A., "First results from a laboratory hypertelescope using single-mode fibers," AEA 477, 345-352 (2008).

[16] Malbet, F., Kern, P., Schanen-Duport, I., Berger, J.-P., Rousselet-Perraut, K., and Benech, P., "Integrated optics for astronomical interferometry. I. Concept and astronomical applications," AE $A S$ 138, 135-145 (July 1999).

[17] Berger, J. P., Rousselet-Perraut, K., Kern, P., Malbet, F., Schanen-Duport, I., Reynaud, F., Haguenauer, P., and Benech, P., "Integrated optics for astronomical interferometry. II. First laboratory white-light interferograms," AEAS 139, 173-177 (1999).

[18] Charles, N., Jovanovic, N., Gross, S., Stewart, P., Norris, B., O’Byrne, J., Lawrence, J. S., Withford, M. J., and Tuthill, P. G., "Design of optically path-length-matched, three-dimensional photonic circuits comprising uniquely routed waveguides," Appl. Opt. 51, 6489 (2012).

[19] Antichi, J., Dohlen, K., Gratton, R. G., Mesa, D., Claudi, R. U., Giro, E., Boccaletti, A., Mouillet, D., Puget, P., and Beuzit, J.-L., "BIGRE: A Low Cross-Talk Integral Field Unit Tailored for Extrasolar Planets Imaging Spectroscopy," ApJ 695, 1042-1057 (2009).

[20] Antichi, J., Rabou, P., Patru, F., Giro, E., Girard, J., and Mourard, D., "The hypertelescope at work with a BIGRE integral field unit," in [SPIE], 8172 (2011).

[21] Jolissaint, L., "Synthetic modeling of astronomical closed loop adaptive optics," Journal of the European Optical Society - Rapid publications, 5, $10055 \mathbf{5}$ (2010).

[22] Rousset, G., Lacombe, F., Puget, P., Hubin, N. N., Gendron, E., Fusco, T., Arsenault, R., Charton, J., Feautrier, P., Gigan, P., Kern, P. Y., Lagrange, A.-M., Madec, P.-Y., Mouillet, D., Rabaud, D., Rabou, P., Stadler, E., and Zins, G., "NAOS, the first AO system of the VLT: on-sky performance," in [SPIE], SPIE 4839, 140-149 (2003).

[23] Beuzit, J.-L., Feldt, M., Dohlen, K., Mouillet, D., Puget, P., Wildi, F., Abe, L., Antichi, J., Baruffolo, A., Baudoz, P., Boccaletti, A., Carbillet, M., Charton, J., Claudi, R., Downing, M., Fabron, C., Feautrier, P., Fedrigo, E., Fusco, T., Gach, J.-L., Gratton, R., Henning, T., Hubin, N., Joos, F., Kasper, M., Langlois, M., Lenzen, R., Moutou, C., Pavlov, A., Petit, C., Pragt, J., Rabou, P., Rigal, F., Roelfsema, R., Rousset, G., Saisse, M., Schmid, H.-M., Stadler, E., Thalmann, C., Turatto, M., Udry, S., Vakili, F., and Waters, R., "SPHERE: a planet finder instrument for the VLT," in $[S P I E], 7014$ (2008). 